\title{
Essais
}

ESSAIS

Revue interdisciplinaire d'Humanités

\section{La querelle des philanthropinistes et néohumanistes : pratiques et débats éducatifs en Allemagne autour de 1800}

Mathilde Lerenard

\section{(2) OpenEdition \\ Journals}

Édition électronique

URL : https://journals.openedition.org/essais/9857

DOI : 10.4000/essais. 9857

ISSN : 2276-0970

Éditeur

École doctorale Montaigne Humanités

\section{Édition imprimée}

Date de publication : 15 avril 2014

Pagination : 81-98

ISBN : 978-2-9544269-2-1

ISSN : $2417-4211$

Référence électronique

Mathilde Lerenard, «La querelle des philanthropinistes et néohumanistes : pratiques et débats éducatifs en Allemagne autour de 1800 », Essais [En ligne], 4 | 2014, mis en ligne le 17 janvier 2022, consulté le 20 janvier 2022. URL : http://journals.openedition.org/essais/9857 ; DOI : https://doi.org/ 10.4000/essais.9857 


\section{La querelle des philanthropinistes et néohumanistes : pratiques et débats éducatifs en Allemagne autour de 1800}

Mathilde Lerenard

En Allemagne la période autour de 1800 est très riche en débats et réformes pédagogiques. On tente de moderniser le système scolaire allemand par des initiatives privées et publiques : pédagogues et hommes d'État se lancent dans diverses réflexions et actions afin de réformer l'éducation des jeunes Allemands. On voit apparaître de nouveaux établissements scolaires expérimentaux, comme les Philanthropines (Philanthropinen). Nombreux sont ceux qui se penchent sur les questions d'éducation, notamment des philosophes et penseurs tels que Kant, Herder, Wilhelm von Humboldt ${ }^{1}$. Les périodiques attestent également l'importance que prend l'éducation dans les débats publics : on trouve de nombreux articles traitant de cette thématique dans les périodiques à forts tirages de l'époque, comme l'Allgemeine Deutsche Bibliothek (1765-1806) de Friedrich Nicolai ou la Berlinische Monatsschrift (1783-96) de Johann Erich Biester et Friedrich Gedike. D’autres périodiques sont créés et dédiés entièrement à ce sujet, comme les Dessauer pädagogische Unterhaltungen (Les discussions pédagogiques de Dessau) (1777-79) de Basedow, l'Allgemeine Revision des gesamten Schul- und Erziehungswesens (Révision de l'intégralité du système éducatif et scolaire) (1785-92) de Campe, ou encore le Braunschweigisches Journal philosophischen, philologischen und pädagogischen Inhalts (Journal philosophique, philologique et pédagogique de Braunschweig) (1788-91) publié par un réseau de pédagogues philanthropinistes. Il y a donc un réel foisonnement d'idées sur le sujet. Cette richesse des débats est due à l'affrontement d'opinions divergentes, qui conduisent parfois à de véritables querelles. Et on peut en effet nettement distinguer deux partis pédagogiques à cette époque en Allemagne : le philanthropinisme et le néohumanisme.

1 Immanuel Kant, Über Pädagogik (Réflexions sur l'éducation), Hermann Holstein (éd.), Bochum, Kamp, 1961, recueil de cours sur la pédagogie ; Johann Gottfried Herder, Schulreden (Discours sur l'école), Leipzig, Reclam, 1903; Humanitätsbriefe, Iéna, Diederichs, 1919; Wilhelm von Humboldt, Königsberger Schulplan (Plan scolaire de Königsberg) et Litauischer Schulplan (Plan scolaire de Lituanie), in Ders, Werke in fünf Bänden, A. Flitner et K. Giel (éd.), Darmstadt, Wissenschaftliche Buchgesellschaft, 1964, vol. 4, p. 168-173. 
L'historiographie ne propose pas encore d'étude comparée de ces deux courants pédagogiques, mais les aborde dans une perspective chronologique, cantonnant le philanthropinisme au XVIII ${ }^{e}$ siècle et le néohumanisme au XIX ${ }^{\mathrm{e}}$ siècle, sans traiter à proprement parler de la période charnière de 1800, durant laquelle les deux idéologies s'affrontent. De plus, ces études mettent systématiquement l'accent sur ce qui oppose ces deux courants, sans aborder ce qui les unit et en oubliant parfois de mettre en regard théories et pratiques effectives ${ }^{2}$. Une étude comparative de ces deux modèles concurrents, prenant en compte aussi bien les théories que les pratiques éducatives, permet de mieux cerner la complexité de la relation qu'entretiennent ces deux courants et vient nuancer la thèse d'un affrontement irréductible entre eux. L'étude d'un cas particulier, celui de Friedrich Gedike et de ses établissements berlinois, s'inscrit dans cette perspective comparative, et se révèle particulièrement intéressante du fait de la place tout à fait originale que ce pédagogue occupe au sein de cette querelle. Dans quelle mesure ces deux modèles éducatifs s'opposent-ils ? Cette opposition est-elle aussi radicale qu'il y paraît ? N'existe-t-il pas au contraire un certain nombre de points communs ou de convergences? Le succès de deux grands lycées berlinois sous la direction de Friedrich Gedike vient démentir la radicalité de cette opposition et l'incompatibilité de ces deux modèles et témoigne d'une tentative de conciliation de courants pédagogiques divergents.

Cette étude comparative s'appuie sur des textes théoriques, parfois polémiques, issus de périodiques ou d'ouvrages sur l'éducation, ainsi que sur des programmes scolaires ou des descriptions du fonctionnement de différents établissements, ou encore sur des manuels scolaires et des sujets d'examens.

\section{Le philanthropinisme et le néohumanisme : deux modèles éducatifs radicalement opposés?}

Le philanthropinisme (Philanthropinismus) est un courant éducatif né en Allemagne dans la seconde moitié du XVIII siècle. Ses partisans se désignent comme " philanthropinistes " (Philanthropinisten) ou "philanthropes " (Philanthropen) et collaborent à la publication de périodiques, grâce auxquels ils font circuler leurs idées et rapportent leurs expériences; ils contribuent aussi à

2 Joachim Detjen, Politische Bildung: Geschichte und Gegenwart in Deutschland, Munich, 2007 ; Notker Hammerstein, Ulrich Hermann (éd.), Vom späten 17. Jahrbundert bis zur Neuordnung Deutschlands um 1800, in Christa Berg (éd.), Handbuch der deutschen Bildungsgeschichte, vol. 2, Munich, Beck, 2005 ; Dietrich Benner, Herwart Kemper (éd.), Die pädagogische Bewegung von der Aufklärung bis zum Neuhumanismus, Weinheim, Beltz, 2003 ; Herwig Blankertz, Die Geschichte der Pädagogik von der Aufklärung bis zur Gegenwart, Wetzlar, Büchse der Pandora,1982 ; Friedrich Paulsen, Geschichte des gelehrten Unterrichts auf den deutschen Schulen und Universitäten vom Ausgang des Mittelalters bis zur Gegenwart, mit besonderer Rücksicht auf den klassischen Unterricht, 2 vol., $3^{e}$ édition augmentée, Leipzig, Veit, 1919-21, reprint Berlin, de Gruyter, 1961. 
la création d'écoles, les "philantropines " (Philanthropin ou Philanthropinum) ${ }^{3}$. Ils se regroupent autour de grandes figures comme Johann Bernhard Basedow (1724-1790), le père du philanthropinisme, Joachim Heinrich Campe (17461818), Christian Gotthilf Salzmann (1744-1811) et Ernst Christian Trapp (1745-1818).

Le terme " néohumanisme " n'est quant à lui introduit qu'en 1885 par l'historien Friedrich Paulsen afin de distinguer le courant pédagogique allemand de l'humanisme de la Renaissance et d'en souligner sa spécificité. Les partisans du néohumanisme forment un mouvement moins distinct et moins visible que le philanthropinisme. Cependant, un philosophe et ministre de l'éducation bavarois de l'époque, Friedrich Immanuel Niethammer (17661848), parle du courant " humaniste " (Humanismus) pour désigner ce qu'on appelle aujourd'hui dans l'historiographie le "néohumanisme " ${ }^{4}$. Il existe donc une certaine conscience de la part des néohumanistes d'appartenir à un courant de pensée, ainsi que l'idée d'une filiation avec la culture antique, avec la paideia grecque - Niethammer emprunte le terme Humanismus à Cicéron : humanitas. Les néohumanistes prônent le retour aux sources gréco-latines et remettent à l'honneur la culture antique.

\section{Une querelle pédagogique}

Des débats animés sur la scène publique allemande

Dans la seconde moitié du XVIII ${ }^{e}$ et les premières décennies du XIX ${ }^{\mathrm{e}}$ siècle, les débats pédagogiques fleurissent dans les journaux. Ces débats sont non seulement nombreux, mais aussi animés et conflictuels, comme le montre l'ouvrage de Friedrich Immanuel Niethammer (1766-1848) intitulé La Querelle du

3 Le premier institut philanthropiniste est créé en 1774 à Dessau. Johann Bernhard Basedow en est le directeur jusqu'en 1776. L'établissement ferme en 1793 en raison de désaccords avec le personnel enseignant et de problèmes d'ordre organisationnel et financier. Une soixantaine de Philanthropines sont créées en Allemagne (Voir à ce propos Christa Berg (éd.), Handbuch der deutschen Bildungsgeschichte, vol. 2, Munich, Beck, 2005).

4 Friedrich Immanuel Niethammer, Der Streit des Philanthropinismus und des Humanismus in der Theorie des Erziehungs-Unterrichts unsrer Zeit, Iéna, Frommann, 1808. Le terme d'humanisme pour désigner la période historique et culturelle est utilisé pour la première fois par Karl Hagen en 1841 (Friedrich Paulsen, Geschichte des gelehrten Unterrichts auf den deutschen Schulen und Universitäten vom Ausgang des Mittelalters bis zur Gegenwart, op. cit.).

5 Philosophe, professeur de philosophie et de théologie de 1794 à 1804 à Iéna; il devient actif dans le domaine de l'éducation (Oberschulkommissar) à partir de 1806 en Bavière, où il réforme le système scolaire selon les conceptions néohumanistes, et devient membre du Conseil de l'éducation (Oberschulrat) et du Conseil ecclésiastique (Oberkirchenrat) bavarois à partir de 1826 (voir Herwig Blankertz, op. cit. ; Michael Schwarzmaier, Friedrich Immanuel Niethammer, ein bayerischer Schulreformator. Teil 1: Niethammers Leben u. Wirken bis zum Jahre 1807, Aalen, Scientia-Verlag, 1974). 
philanthropinisme et de l'humanisme dans la théorie de l'enseignement de notre temps et publié en 1808, une véritable diatribe contre le philanthropinisme. Cet ouvrage est intéressant à plus d'un titre : d'une part, parce qu'il adopte une démarche comparative, négligée par la recherche sur l'histoire de l'éducation, et, d'autre part, parce qu'il témoigne du caractère passionné et polémique des affrontements entre les deux systèmes éducatifs qui tentent de s'imposer dans le paysage scolaire allemand. Il souligne aussi que ces deux modèles pédagogiques sont contemporains : ils ne se succèdent pas uniquement dans le temps, mais s'affrontent pendant un certain nombre d'années sur un plan théorique et pratique.

\section{L'ouvrage de F. I. Niethammer}

Dans cet ouvrage, l'auteur oppose de manière systématique le philanthropinisme et l'humanisme. Il expose les fins et les moyens de ces deux courants point par point, en les opposant de manière symétrique. Cette opposition est d'ailleurs visible dans la présentation même de l'ouvrage : un chapitre est présenté sous forme de tableau composé de deux colonnes, l'une dédiée au philanthropinisme et l'autre au néohumanisme. L'auteur use - et abuse - de tous les moyens rhétoriques pour démontrer cette opposition, en jouant par exemple sur les antonymes et en multipliant les jeux de miroir, faisant apparaittre les deux courants comme deux images inversées. Il déclare ainsi à propos du néohumanisme : «Il ne s'agit pas pendant le cours d'accumuler certaines connaissances, mais bien plutôt d'exercer l'esprit ", et au sujet du camp adverse : "Il ne s'agit pas pendant le cours d'exercer l'esprit pour lui-même, mais bien plutôt de le remplir du plus grand nombre possible de connaissances utiles $"^{6}$. Il va jusqu'à affirmer que l'humanisme est une "éducation à l'humanité " (Bildung zur Humanität) et le philanthropinisme une " éducation à la bestialité » (Bildung zur Bestialität).

Niethammer insiste également en permanence sur la radicalité de l'opposition de ces deux courants. Les termes de dispute ou querelle ponctuent sa démonstration : il parle de " la dispute des deux extrêmes opposés de la pédagogie ", de "la querelle des deux systèmes éducatifs opposés ", de " point de discorde" (Streitpunkt). La métaphore du combat fait figure de leitmotiv, on a le sentiment en le lisant qu'une véritable guerre a éclaté : "le combat qui a éclaté de nos jours »" déclare-t-il pour désigner cette querelle pédagogique.

6 Friedrich Immanuel Niethammer, Der Streit des Philanthropinismus und des Humanismus in der Theorie des Erziehungs-Unterrichts unsrer Zeit, Iéna, Frommann, 1808 (Dritter Abschnitt: Von den Grundsätzen des Erziehungsunterrichts im Allgemeinen) : «Es kommt bei dem Erziehungsunterricht nicht sowohl darauf an, bestimmte Kenntnisse zu sammeln, als vielmehr darauf, den Geist zu üben "; "Es kommt bei dem Erziehungsunterricht nicht sowohl darauf an, den Geist an und für sich zu üben, als vielmehr darauf, ihn mit der möglichst grössten Masse brauchbarer Kenntnisse auszurüsten ».

7 Ibid., Introduction, p. 3-8 : « [Streit] der beiden entgegengesetzten Extreme der Pädagogik » 
Niethammer définit ainsi le néohumanisme en stricte opposition par rapport au nouveau courant pédagogique né en Allemagne dans les années 1770 qu’est le philanthropinisme. Il se livre à une véritable attaque contre ces nouvelles idées et méthodes, qu'il faut selon lui endiguer et faire disparaitre au plus vite.

\section{Des conceptions opposées}

Philanthropinistes et néohumanistes s'opposent sur plusieurs fronts en matière de pédagogie, et prennent même bien souvent des directions radicalement opposées.

\section{Les fins éducatives}

Le premier front sur lequel les deux camps pédagogiques s'affrontent est celui des fins qu'ils assignent à l'enseignement. Alors que les néohumanistes envisagent la formation de l'individu comme une fin en soi, comme le développement de l'humanité en l'homme, c'est-à-dire de toutes les facultés humaines de manière harmonieuse, les philanthropinistes voient en l'éducation un moyen d'intégration de l'individu dans la société : il s'agit pour ces derniers de former l'homme au rôle qu'il jouera dans le monde en fonction de son rang social et de son métier $^{8}$. On a donc, d'un côté, les tenants d'une formation générale, universelle, qui développerait l'humanité en l'individu, et, de l'autre, les partisans d'une formation avant tout utile et spécifique en fonction du rang social et du métier auquel se destine l'individu. Les néohumanistes placent ainsi l'homme, l'individu en tant qu'être humain et membre de l'humanité, au centre de leurs préoccupations, tandis que les philanthropinistes s'intéressent principalement au citoyen, à l'individu comme membre d'une communauté civile.

L'objectif d'une "formation universelle de l'homme " (allgemeine Menschenbildung) ${ }^{9}$ est en effet au centre des préoccupations de Wilhelm von Humboldt (1767-1835). Ce qu'il vise dans ses plans éducatifs ${ }^{10}$, c'est le déve-

et « streit der beiden entgegengesetzten Unterrichtssysteme »; « das lebhafte Interesse, das sich für die Entscheidung jenes Streitpunktes gezeigt hat "; "der Streit [...] der sich in unsern Tagen zum Ausbruch entzündet hat. »

8 Le but de l'éducation néohumaniste est " la formation universelle de l'homme ", il s'agit d'une « fin en soi » (« einen eigenen für sich bestehenden Zweck, die allgemeine Bildung des Menschen »), tandis que le but de l'éducation philanthropiniste est " la formation de l'homme à sa future destinée dans le monde " (" keinen eigenen für sich bestehenden, sondern nur relativen Zweck, die Bildung des Menschen für seine künftige Bestimmung in der Welt ») (Friedrich Immanuel Niethammer, op. cit., p. 18).

9 "Litauischer Schulplan ", in W. v. Humboldt, Gesammelte Schriften, Berlin, 1920, vol. 13, p. 276-277.

10 Le Plan éducatif de Königsberg (Königsberger Schulplan) et celui de Lituanie (Litauischer Schulplan), tous deux rédigés en 1809. 
loppement harmonieux des forces de l'individu vers un tout, vers l'humanité ; il veut donner à chaque enfant la chance de développer en lui son " humanité ", et non pas le former immédiatement ou de façon précoce à un métier : "Toutes les écoles qui ne sont pas dédiées à un ordre en particulier mais à la nation entière ou à l'État doivent viser uniquement la formation universelle de l'homme. Tout ce que le besoin de la vie ou d'un de ses métiers exige doit être séparé et acquis une fois l'enseignement général terminé. Si on mélange les deux [ces deux types d'enseignement : la formation professionnelle et la formation générale], l'éducation devient impure, et on n'obtient ni des hommes complets ni des citoyens complets dans les différentes classes ${ }^{11}$.

Les philanthropinistes quant à eux ont un souci pratique, mais aussi moral d'harmonie sociale : l'individu doit être un membre utile de la société et contribuer à son progrès. L'article de Bahrt dans la Révision générale intitulé "Du but de l'éducation $~ "^{12}$, véritable manifeste du projet philanthropiniste, le montre clairement : le but de l'éducation est le bonheur de l'homme, ce dernier étant défini comme la joie de l'activité : "Toute l'éducation de l'homme repose sur le principe de l'habituer à l'activité, à l'usage de toutes ses forces, car tout plaisir, toute joie a pour source l'activité ». En effet, «les joies de la bienfaisance et de l'utilité publique sont parmi les joies humaines les plus douces, [...] les plus pures [...], les plus durables [...], et les plus nobles [...] et enfin les joies les plus parfaites de la vie humaine ". "Qu'il [l'élève] apprenne à trouver dans l'amour, c'est-à-dire dans la représentation de la joie et de la satisfaction qu'il aura procurées à autrui, sa propre joie et sa joie la plus grande ", "qu'il fasse tout pour contribuer au bien public, qu'il apprenne tout dans le but d'être utile $[\ldots] »^{13}$.

11 Traduit de W. v. Humboldt, Gesammelte Schriften, Berlin, 1920, Bd. XIII, p. 276-277 : «Alle Schulen aber, deren sich nicht ein einzelner Stand, sondern die ganze Nation oder der Staat für diese annimmt, müssen nur allgemeine Menschenbildung bezwecken. Was das Bedürfnis des Lebens oder eines einzelnen seiner Gewerbe erheischt, muß abgesondert und nach vollendetem allgemeinen Unterricht erworben werden. Wird beides vermischt, so wird die Bildung unrein, und man erhält weder vollständige Menschen noch vollständige Bürger einzelner Klassen. »(Der litauische Schulplan).

12 Karl Friedrich Bahrt, "Über den Zweck der Erziehung (1785), in Campe, Joachim Heinrich (éd.), Allgemeine Revision des gesammten Schul- und Erziehungswesens, vol. 1, 1785, p. 1-124.

13 Ibid., p. 45-46 : "Thätigkeit, oder Leben im wahren Sinn, ist - Freude: ist die einzige Quelle aller angenehmen Empfindungen. "; "Thätigkeit (nehmlich von aussen her zwanglose, d.h. freye und dem Grade der Kräfte angemessene Unthätigkeit) oder Leben ist - was die Griechen Hedone nannten - Vergnügen. Das vollkommenste Leben, das angenehmste Gefühl ist bei dem Leben, oder den Thätigkeiten des Geistes: das unvollkommste bei der bloßen Körperbewegung. "; " dass alles bei der Erziehung des Menschen darauf beruht, dass ich ihn an Thätigkeit, an den Gebrauch aller seiner Kräfte gewöhne, weil alles Vergnügen, aller Freudegenuss in Thätigkeit besteht. "; " die Freuden des Wohlthuns und Nützlichwerdens sind unter allen Menschenfreuden die süssesten in der Empfindung, [...] die reinsten [...], die dauerhaftesten [...] die edelsten [...] die vollkommensten Freuden des menschlichen Lebens "; " dass er in der Liebe d.h. in der Vorstellung der von ihm bewirkten Freude und 
La perspective adoptée par les philanthropistes est ainsi avant tout utilitariste, et vise le bien-être de la communauté, tandis que les néohumanistes centrent leurs réflexions sur l'individu et l'épanouissement universel et harmonieux de ses facultés.

\section{Les méthodes éducatives}

Le deuxième terrain d'affrontement entre les deux courants éducatifs concurrentiels est celui des méthodes d'enseignement. Alors que les néohumanistes s'attachent à un enseignement purement linguistique et à un savoir livresque (étude de la littérature antique), les philanthropinistes se tournent vers un enseignement scientifique et expérimental (l'algèbre et la géométrie, les sciences naturelles, les nouvelles découvertes), sans abandonner cependant tout enseignement linguistique. Ainsi Basedow et ses collaborateurs mettentils l'accent sur un enseignement tourné vers la pratique et un apprentissage par les sens. Les élèves doivent être amenés à observer, manipuler et tirer eux-mêmes les conclusions de leurs observations et expériences. On installe des cabinets de sciences naturelles ou des salles expérimentales de sciences physiques dans les philanthropines et on organise des " classes vertes " pour les élèves. Les philanthropinistes entendent ainsi opposer à l'enseignement des mots et par les mots un enseignement des choses par les choses. Ils méprisent d'ailleurs les mots, qu'ils considèrent comme des coquilles vides. Le philanthropiniste Trapp, un des collaborateurs de la Révision générale, part du principe que les connaissances linguistiques n'accroissent pas les connaissances scientifiques (Sachkenntnisse) et que l'apprentissage des langues n'est qu' " un mal nécessaire » : "La langue est constituée de signes des idées. Une idée a besoin de signes pour être saisie ou communiquée. Cent signes pour une idée ne sont pas cent nouvelles idées. Si je sais réciter le Notre Père en cent langues, ce n'est pas pour autant que j'en comprends mieux le sens. La diversité des langues permet par conséquent aussi l'apprentissage de nombreuses langues, mais pas l'accroissement quantitatif et qualitatif des idées. Celle-ci [l'idée] est bien plutôt gênée par celle-là [la langue]. Car le temps et la force qui sont utilisés à l'apprentissage de celles-ci [des langues] doivent être soustraits à l'accroissement du nombre d'idées $»^{14}$. Pour les philanthropinistes, les enfants doivent apprendre à connaître le monde dans lequel ils vivent, non pas à travers les mots, mais par une confrontation directe à celui-ci, par leurs propres expériences, par les sens et la réflexion.

Zufriedenheit Andrer seine eigene und höchste Freude finden lerne »; "- dass er alles thue, um wohlzuthun: - dass er alles lerne, um zu nützen. "

14 Ernst Christian Trapp, "Über den Unterricht in Sprachen ", in Allgemeine Revision, op. cit., Jahrgang 4, 1788, Heft 11. 


\section{Les contenus enseignés}

Enfin, le troisième front sur lequel s'affrontent philanthropinistes et néohumanistes est celui des contenus enseignés.

Comme l'humanisme de la Renaissance, l'enseignement néohumaniste se caractérise par une étude intensive et approfondie de la littérature grecque et romaine. L'importance donnée à l'enseignement linguistique, et tout particulièrement celui des langues anciennes, découle de l'idéal néohumaniste, selon lequel la parole est ce qui distingue l'homme de l'animal, et la parole cultivée et soignée ce qui distingue l'homme - pleinement humain - du barbare et qui offre l'accès au spirituel et au divin. Cet enseignement essentiellement linguistique est centré sur les langues anciennes (le latin et le grec), l'Antiquité étant considérée comme le modèle de civilisation par excellence. Wilhelm von Humboldt définit ainsi dans son Étude de l'Antiquité et du Grec en particulier la langue et la culture grecques comme la voie censée conduire à une humanité idéale ${ }^{15}$.

L'enseignement philanthropiniste se tourne au contraire résolument vers le présent, la modernité et l'utilité. La prédominance de l'étude des langues anciennes dans les écoles est sévèrement critiquée et jugée absurde par les philanthropinistes ; elle doit céder la place à l'étude des langues modernes, comme le français, l'anglais et l'italien, et surtout à l'étude de la langue maternelle, l'allemand. L'apprentissage des langues modernes est en effet jugé indispensable au développement des échanges commerciaux et scientifiques, et donc nécessaire aux futures activités professionnelles des jeunes élèves ${ }^{16}$.

Autant en ce qui concerne les objectifs que les méthodes d'enseignement et les contenus enseignés, les philanthropinistes et les néohumanistes représentent deux pôles antagonistes. C'est sur cette relation d'opposition qu'insiste l'historiographie. Cependant, si on s'intéresse de plus près aux personnes, aux philosophes et pédagogues philanthropinistes et néohumanistes, à leur collaboration, à la communication entre eux et à ce qui est mis concrètement en place dans

15 Wilhelm von Humboldt, De l'étude de l'Antiquité et du Grec en particulier (Über das Studium des Alterthums und des Griechischen insbesondere), écrit en 1793, publié pour la première fois dans Albert Leitzmann (éd.), Sechs ungedruckte Aufsätze über das klassische Altertum von Wilhelm von Humboldt, Leipzig, 1896.

16 Le philanthropiniste Trapp décrit l'apprentissage des langues modernes comme un mal nécessaire, qui permet cependant la communication scientifique entre les nations, que ce soit dans le domaine des sciences dures (médecine, biologie, physique, astronomie...) ou des sciences humaines et politiques (philosophie, géographie, droit...) : «L'apprentissage des langues étrangères est donc un mal nécessaire, nécessaire en raison des relations nécessaires des nations entre elles, qui ne se réduit pas simplement au commerce et aux mutations de ce genre, mais signifie aussi communication de connaissances. " ("Erlernung fremder Sprachen ist also ein notwendiges Übel, notwendig wegen des notwendigen Verkehrs der Nationen mit einander, welches nicht bloß Handel und Wandel dergl., sondern auch Mitteilung von Kenntnissen bedeutet. » (Ernst Christian Trapp, Revisionswerk, op. cit., vol. 9, p. 216) 
les établissements scolaires, on peut se demander s'il ne faut pas relativiser cette représentation. Ces deux courants pédagogiques concurrents ont en effet un certain nombre de similitudes et de points de convergence non négligeables.

\section{Les philanthropinistes et les néohumanistes se font-ils la guerre ou mènent-ils un combat commun?}

\section{Un ennemi commun : le système scolaire allemand de l'époque}

Philanthropinistes et néohumanistes sont peut-être moins opposés qu'il n'y paraît au premier abord ; ils ont avant tout un ennemi commun : le système scolaire allemand tel qu'il existe à l'époque. Et pour les pédagogues des deux camps, c'est celui-ci qu'il faut combattre et réformer. Cet enseignement est resté identique des siècles durant : un enseignement de la religion et du latin essentiellement, fondé sur un apprentissage par cour. Les rapports de l'Oberschulkollegium ${ }^{17}$ (Conseil supérieur de l'éducation de Prusse, créé en 1787) dévoilent ainsi que la grande majorité des heures d'enseignement dispensées dans les écoles des villes prussiennes est consacrée à l'apprentissage du latin, tandis qu’à la campagne on enseigne principalement le catéchisme.

\section{Des objectifs communs - au-delà des antagonismes}

\section{Le développement de la raison (Verstand et Vernunft)}

Ces deux concepts éducatifs concurrents, si radicalement opposés sur un certain nombre de points, mènent pourtant un combat commun contre l'apprentissage par cœur, dénué de toute réflexion personnelle. Tous deux ont pour ambition de développer l'entendement et l'esprit critique, et s'évertuent à le faire à travers leur enseignement respectif.

Wilhelm von Humboldt donne ainsi la priorité à l'apprentissage de la réflexion : il faut apprendre aux élèves à apprendre ${ }^{18}$ et à penser. L'école a le devoir de guider les jeunes gens vers la Mündigkeit ${ }^{19}$, c'est-à-dire vers une réflexion autonome. De même, le philanthropiniste Basedow veut faire de ses élèves de l'institut de Dessau des hommes " ayant atteint une majorité

17 Ces rapports sont consultables au Geheimes Staatsarchiv à Berlin-Dahlem ; une partie est également retranscrite dans l'ouvrage de Paul Schwartz, Die Gelehrtenschulen Preussens unter dem Oberschulkollegium (1787-1806), in Monumenta Germaniae Paedagogica, hrsg. von der Gesellschaft für deutsche Erziehungs- und Schulgeschichte, Berlin, Weidmannsche Buchhandlung, 1912.

18 Voir Ulrike Heuer, Ruth Siebers (éd.), Weiterbildung am Beginn des 21. Jahrhunderts: Festschrift für Wiltrud Gieseke, Münster, Waxmann, 2007, p. 37-38.

19 Le concept de Mündigkeit désigne en allemand la majorité ou la maturité intellectuelle de l'individu, sa capacité à penser librement et de manière autonome. On le trouve par exemple chez Immanuel Kant, Qu'est-ce que les Lumières?, 1784. 
intellectuelle "(" mündige Männer »). On retrouve donc cette notion de " majorité intellectuelle" (Mündigkeit) dans les deux courants. Le philanthropiniste Bahrt développe cette idée dans le premier article de la Révision générale: l'élève doit " apprendre la vérité à partir de préconnaissances élémentaires, et se l'approprier ensuite par sa réflexion autonome - ce dont l'individu que l'on habitue à tout accepter par fidélité et croyance est incapable »; il ajoute : " [que l'élève] s'exerce de bonne heure à l'usage des forces nécessaires à cette connaissance que sont l'attention, la réflexion, l'abstraction etc., que l'enseignant le guide sur la voie d'une pensée raisonnée $[\ldots] »^{20}$.

\section{La recherche du bonheur (Glückseligkeit)}

En outre, philanthropinistes et néohumanistes affirment poursuivre le même but : le bonheur - bien que le chemin emprunté pour y arriver ne soit pas le même. Chez les néohumanistes, le bonheur correspond au degré le plus haut de développement des facultés et de la personnalité de l'homme ; mais c'est grâce à cet épanouissement individuel que l'homme est capable de contribuer au bien de l'humanité. Chez les philanthropinistes, c'est la contribution au bien commun qui rend heureux, comme les extraits de l'article de Bahrt cité plus haut le montrent clairement. On aboutit finalement au même résultat : le bonheur de l'individu et de l'humanité - en suivant toutefois un parcours inverse.

\section{Des références communes : les Lumières et la Grèce antique}

Les Lumières, avec leur idée de progrès de l'humanité dans l'histoire, et leur postulat selon lequel le progrès se ferait grâce à l'éducation, constituent la référence commune des néohumanistes et des philanthropinistes. Ce qui caractérise les tenants des deux camps pédagogiques, c'est leur même foi inébranlable en l'éducation : c'est elle qui fait de l'homme un homme ; elle est considérée comme vecteur d'humanité. Sans elle, l'homme n'est et ne reste qu'un animal. En outre, la "majorité » de l'homme (la Mündigkeit), la réflexion autonome, représente l'idéal et le projet des Lumières. Ce projet est adopté aussi bien par les philanthropinistes que par les néohumanistes.

Enfin, les partisans des deux courants se réfèrent au même modèle : la Grèce antique du siècle de Périclès. Chez les néohumanistes, c’est le citoyen athénien qui représente le plus haut degré de réalisation de l'humanité en

20 Karl Friedrich Barth, «Über den Zweck der Erziehung », op. cit., p. 30 : « dass er Wahrheit bloss aus diesen Vorkenntnissen in elementarischer Ordnung erlerne, und folglich durch Selbstdenken sie zum Eigenthum seiner Seele mache, was sie bei keinem Menschen ist, der gewöhnt wird, sie auf Treu und Glauben anzunehmen - dass er früh die zu dieser Kenntnis nöthigen Kräfte der Attention, der Reflexion, der Abstraktion u.s.w. übe, und ihn zum vernünftigen Denken anleite: - dass er ihn an dem Auffinden der Wahrheiten Freude finden lehre. » 
l'homme. Mais le modèle de la Grèce antique est aussi présent chez les philanthropinistes, avec par exemple la figure de Socrate, modèle de l'enseignant avec son art d'accoucher les esprits, la maïeutique. Les philanthropinistes se réferrent également aux habitants de Sparte : dans les philanthropines, les élèves pratiquent des activités en pleine nature. Courses d'orientation dans la forêt, traversées de lacs à la nage ou encore saut d'obstacles figurent au programme d'éducation physique. Le courage est mis en avant et récompensé et les vainqueurs se voient attribuer une couronne de lauriers ${ }^{21}$.

Au-delà des antagonismes évidents et réels, il faut donc relativiser la relation de stricte opposition entre le philanthropinisme et le néohumanisme. Ces deux courants pédagogiques ont une même foi en l'éducation, des objectifs éducatifs, un projet commun, celui des Lumières, et des références ou modèles communs.

En outre, ces deux concepts éducatifs ne sont pas incompatibles, comme le montre l'exemple du pédagogue berlinois Friedrich Gedike.

\section{Une tentative de conciliation : le projet et l'action du pédagogue berlinois Friedrich Gedike - une savante synthèse entre philanthropinisme et néohumanisme}

Friedrich Gedike, né en 1754 et mort en 1803, est enseignant et directeur de deux établissements scolaires publics à Berlin, mais aussi auteur de manuels scolaires et de nombreux ouvrages traitant de pédagogie, ainsi que d'articles parus dans des périodiques dédiés à l'éducation. Il est également co-éditeur de la revue des Lumières, la Berlinische Monatsschrift, membre du Consistoire et du Conseil d'Éducation de Prusse, membre de l'Académie des Arts et de l'Académie des Sciences de Berlin, membre de différentes sociétés secrètes (le Montagsclub et la Mittwochsgesellschaft) et franc-maçon. Gedike est donc très présent sur la scène intellectuelle berlinoise et au-delà, et il est intéressant en raison de sa double activité de théoricien et de praticien de l'éducation : il est à la fois un homme de lettres et un pédagogue engagé et très actif, influent au sein des sphères du pouvoir.

Dans l'histoire de l'éducation, et au sein des débats pédagogiques de l'époque, Gedike occupe une place originale. Dans l'historiographie, il est souvent désigné comme " néohumaniste ». Wilhelm Dilthey le présente même comme " le père

21 Ces activités physiques sont par exemple pratiquées au Philanthropinum de Schnepfenthal. Salzmann, le fondateur et directeur de cet institut philanthropiniste, le décrit dans Über die Erziehungsanstalt zu Schnepfenthal [Sur la maison d'éducation de Schnepfenthal], Schnepfenthal, 1808 . 
du néohumanisme prussien $»^{22}$, tout comme Harald Scholtz dans son article "Friedrich Gedike, pédagogue, publiciste et homme politique " ${ }^{23}$. Pourtant, Gedike entretient de nombreux liens avec les philanthropinistes, comme le montrent sa correspondance ainsi que sa collaboration aux périodiques publiés par les philanthropinistes. Il compte en effet parmi les collaborateurs de la Révision générale de la totalité du système scolaire et éducatif ${ }^{4}$. Comment situer alors ce pédagogue berlinois au sein des débats et réformes pédagogiques qui opposent néohumanistes et philanthropinistes?

\section{Friedrich Gedike : une figure de conciliateur}

Friedrich Gedike apparaît comme une figure de conciliateur ou de médiateur entre ces deux courants pédagogiques rivaux. C'est ce qui ressort de l'étude des programmes scolaires de ses deux établissements, ainsi que des théories éducatives qu'il développe.

Il se présente en homme réfléchi et érudit, qui connaît très bien ces deux courants, et souhaite mettre un terme aux excès ou erreurs de l'un et de l'autre, tout en gardant ce qui lui semble bon, jetant ainsi un regard critique sur ses contemporains et sur l'histoire de l'éducation en Allemagne. Selon lui, on ne cesse de passer d'un extrême à l'autre en matière de pédagogie : "On a renversé ici et là la monarchie despotique des langues anciennes pour mettre les sciences sur le trône $"^{25}$. Les humanistes, mais aussi l'enseignement tel qu'il est pratiqué dans la plupart des écoles publiques en Allemagne au $\mathrm{XVIII}^{\mathrm{e}}$ siècle, qui ne jurent que par les langues anciennes, tout comme les philanthropinistes qui privilégient les sciences, sont rejetés et qualifiés de tyrans par Gedike. Ce dernier tente d'éviter ces deux extrêmes ou despotismes pédagogiques, en cherchant un équilibre entre l'enseignement des langues et des sciences. Il se pose donc bien en figure de médiateur entre deux courants pédagogiques qu’il juge " extrêmes » et « despotiques ".

22 «Der Begründer des preussischen Neuhumanismus» (Harald Scholtz, «Das Friedrichswerdersche Gymnasium in Berlin als Modellschule der Spätaufklärung ", in Benno Schmoldt (éd.), Schule in Berlin - Gestern und Heute, Berlin, Colloquium Verlag, 1989).

23 Harald Scholtz, "Friedrich Gedike, der Schulpädagoge, Publizist und Bildungspolitiker ", in Frank Tosch, Friedrich Gedike (1754-1803) und das moderne Gymnasium, Berlin, Weidler, 2007, p. 31-43. De même, dans son article «Friedrich Gedike (1754-1803). Ein Wegbereiter der preußischen Reform des Bildungswesens ", in Jahrbuch für die Geschichte Mittel- und Ostdeutschlands, vol. 13-14, 1965, p. 128-181.

24 Allgemeine Revision des gesamten Schul-und Erziehungswesens (Voir notre paragraphe introductif).

25 Friedrich Gedike, «Über die Verbindung des wissenschaftlichen und philologischen Unterrichts » (1780), in Gesammlete Schulschriften, Berlin, Unger, vol. 1, 1789. 
Les fins pédagogiques poursuivies par Gedike : recherche d'un juste milieu entre les objectifs des philanthropinistes et des néohumanistes

Au-delà de la posture qu'il adopte, les fins qu'il assigne à l'éducation sont à la fois fidèles aux principes néohumanistes et philanthropinistes. Léducation doit contribuer « au bien-être futur de l'humanité $e t$ de l'État ${ }^{26}$. En portant son attention aussi bien sur l'État que sur l'humanité, Gedike ouvre une troisième voie dans le champ de l'éducation, une voie médiane entre celles choisies par les deux courants rivaux. Pour lui, il ne faut négliger aucune de ces deux préoccupations, ni favoriser l'une par rapport à l'autre. On retrouve toujours cette articulation entre l'homme et le citoyen, l'humanité et la communauté civile. Gedike voit d'ailleurs en ses élèves à la fois de "futurs citoyens " et "l'humanité en devenir ${ }^{27}$. Il ajoute, acquiesçant à la vision philanthropiniste, que « se soucier du bien-être des écoles " c'est " se soucier du bien-être de l'État ", et, allant dans le sens des néohumanistes, que l'enseignant a le devoir de former la jeunesse "pour le bien-être de la société humaine ${ }^{28}$. Les enseignants sont autant des éducateurs du genre humain qui guident les élèves sur la voie de l'humanité que les formateurs des citoyens d'un État donné.

\section{L'action de Gedike : un savant équilibre entre philanthropinisme et néohumanisme}

Non seulement la posture adoptée par Gedike et ses fins éducatives font de lui une figure de médiateur entre le concept éducatif néohumaniste et le philanthropinisme, mais c'est aussi toute l'entreprise du pédagogue berlinois qui vise la réalisation d'une véritable synthèse entre ces deux courants. Les discours d'invitation aux examens publics ou aux fêtes annuelles de ses établissements sont une véritable mine d'informations concrètes et précises sur les disciplines enseignées, les supports pédagogiques et le fonctionnement des établissements dirigés successivement par Friedrich Gedike de 1779 à 1803 (le Friedrichs-Werdersches Gymnasium et le Berlinisches Gymnasium zum Grauen Kloster), et témoignent des réformes d'inspiration philanthropiste et néohumaniste mises en place dans les écoles de Gedike.

26 «Zum künftigen Wohl der Menschheit und des Staats " (Friedrich Gedike, Jubelrede (1787), ibid., p. 480.

27 "Er sieht die künftigen Staatsbürger vor sich » (ibid., p. 482) ; " die werdende Menschheit » (ibid., p. 480).

28 "Sorge für den Wohlstand der Schulen ist zugleich Sorge für das Wohl des Staates " (ibid., p. 488) ; « zum Wohl der menschlichen Gesellschaft» (ibid., p. 483). 


\section{Enseignements linguistiques et scientifiques}

L'étude des programmes scolaires et des textes théoriques de Gedike dévoile la recherche constante d'un équilibre entre un enseignement linguistique, préconisé par les néohumanistes, et un enseignement scientifique, dominant chez les philanthropinistes. Ainsi, en recoupant les informations contenues dans les discours d'invitation ${ }^{29}$ du directeur Gedike, on obtient 36 heures de cours au total pour un Primaner, élève de "terminale " (dernière année de lycée), dont 18 heures d'enseignement en langues anciennes et modernes. On a donc bien un équilibre, en termes d'horaires, entre l'enseignement des langues et les leçons de choses.

\section{Langues anciennes et langues modernes}

En ce qui concerne l'enseignement des langues en particulier, point de querelle entre philanthropinistes et néohumanistes, les uns ne jurant que par les langues anciennes, les autres par les langues modernes, on retrouve là aussi chez Gedike cette recherche d'équilibre. Un Primaner du Gymnasium zum Grauen Kloster compte en effet dans son emploi du temps 4 heures hebdomadaires de latin, 4 heures de grec ancien (et 2 heures d'hébreu s'il se destine à une carrière ecclésiastique), donc 8 à 10 heures de langues anciennes par semaine, ainsi que 2 heures d'allemand, 4 de français et 4 d'italien ou d'anglais, soit un total de 10 heures de langues modernes hebdomadaires.

Friedrich Gedike considère en outre, comme les néohumanistes, que l'apprentissage des langues anciennes a une valeur formelle ; il permet de former l'esprit humain, de développer les différentes facultés intellectuelles de l'homme : "Aucune étude n'est aussi habile à éveiller et stimuler toutes les forces de l'esprit qui sommeillent, aucune aussi habile à préparer et former l'âme à toutes les sciences possibles que celle-ci [l'étude des langues anciennes], si elle est conduite selon les règles de l'art et selon une approche philologique $»^{30}$.

Parallèlement, il accorde une grande importance à l'apprentissage des langues modernes, chargé de former des cosmopolites (Weltbürger). La maîtrise des langues vivantes permet de faciliter les échanges culturels et scientifiques avec l'étranger (ici entre l'Allemagne et la France, l'Italie, l'Angleterre et la

29 Ces discours sont réunis en deux volumes intitulés Gesammlete Schulschriften (publiés en 1789 et 1795) et consultables aux archives à la Staatsbibliothek de Berlin.

30 "[...], dass kein einziges Studium so geschickt ist, alle schlummernden Geisteskräfte zu wekken und zu spornen, kein einziges so geschickt, die Seele zu allen möglichen Wissenschaften so vorzubereiten und auszubilden, als eben dieses [das Studium der alten Sprachen], wenn es auf eine philologische und den Regeln der wahren Methodik angemessene Art betrieben wird. Die größten Gelehrten in allen Fächern waren zugleich immer mehr oder weniger gute Humanisten. " (Friedrich Gedike, Über die Verbindung des wissenschaftlichen und philologischen Schulunterrichts, op. cit., p. 23) 
Pologne) ; c'est ce que vise Gedike en insistant sur l'objectif de communication de l'apprentissage des langues modernes ${ }^{31}$. Les intérêts économiques de la Prusse ne sont pas absents de ses réflexions : il juge ainsi l'apprentissage du polonais utile au développement du commerce avec la Pologne et introduit cette option dans le programme du Gymnasium zum Grauen Kloster en 1797.

En outre, une nouvelle valeur et un nouveau rôle sont accordés à l'apprentissage des langues anciennes, harmonisant ainsi les principes pédagogiques des deux courants. L'exemple de l'enseignement du latin est significatif. Selon Gedike, l'apprentissage des langues anciennes a entièrement sa place à l'école, car même s'il n'est pas d'une utilité immédiate dans le quotidien des élèves et leur vie d'adultes, il permet de former aussi bien l'esprit que le goût des élèves et de les familiariser avec les idées édifiantes des Anciens. Il est comme chez les néohumanistes la base de toute formation professionnelle ultérieure. Néanmoins, Friedrich Gedike voit dans l'enseignement du latin un moyen d'apporter aux élèves des connaissances concrètes et pratiques et non pas seulement linguistiques, comme des " concepts moraux, historiques, géographiques et d'histoire naturelle $»^{32}$. Les manuels de latin de Gedike traduisent cette orientation vers une utilité pratique de l'apprentissage de la langue, dans le sens où les élèves y puisent des connaissances pratiques et y exercent leur esprit critique. Ils travaillent à partir d'extraits d'auteurs latins soigneusement sélectionnés par Gedike et ne se contentent pas d'étudier la forme (grammaire, lexique, style), puisqu'une partie du cours est consacrée à l'interprétation du sens des textes. Gedike repense donc entièrement l'apprentissage du latin à l'école et introduit une nouvelle méthode qui connaitra un grand succès durant tout le XIX ${ }^{e}$ siècle : "On a longtemps commencé son enseignement [du latin] à l'envers, de façon tout à fait insensée, par la grammaire. On soumettait [aux élèves] du vocabulaire, des formules sans lien cohérent entre elles et des sentences décousues. J'ai toujours eu la conviction que seuls des récits et des descriptions ont leur place dans un ouvrage pour débutants. Seuls ceux-ci me semblent être compréhensibles et distrayants pour le débutant gouverné par ses sens. Les abstractions l'épuisent. Le fait d'être compréhensible, la simplicité, l'intérêt pratique et la variété furent les règles que je me suis prescrites lors de la compilation de mes recueils de grec, de latin et de français ${ }^{33}$. Ces propos montrent bien que, même si Gedike se range du côté

31 Gedike répète cette idée dans les préfaces de ses manuels de langues vivantes : Französisches Lesebuch (Berlin, 1785), Englisches Lesebuch (2édition augmentée, Berlin, 1797).

32 "Die [...] moralischen, historischen, geographischen, und besonders naturhistorischen Begriffe " (Friedrich Gedike, Praktischer Beitrag zur Methodik des öffentlichen Unterrichts (1781), in F. G., Gesammlete Schulschriften, vol. 1, 1785, p. 127).

33 "Lange hat man den Unterricht höchst widersinnig und verkehrt mit der Grammatik angefangen. Dazu bot man Vokabeln, unzusammenhängende Formeln und abgerissene Sentenzen. Ich habe immer geglaubt, dass in ein Lehrbuch für Anfänger nur Erzählungen und Beschreibungen 
des néohumanistes quant à la place qu'il accorde à l'enseignement du latin dans la formation des élèves, sa méthode d'apprentissage du latin, pensée et mise en pratique dans ses établissements, intègre quant à elle largement des principes philanthropinistes, comme par exemple l'attention portée à la psychologie de l'enfant, ainsi que l'orientation pratique et utilitariste.

\section{Monde antique et monde contemporain}

Un autre aspect de l'originalité de l'enseignement dans les établissements de Gedike est la conjugaison de l'ancien et du moderne. La lecture des Anciens et l'étude de la culture antique y gardent une place importante, mais le monde contemporain n'est pas pour autant laissé de côté : une heure de lecture et de commentaire des journaux est prévue dans l'emploi du temps des élèves et de nombreux sujets d'examens portent sur l'histoire moderne ou sur des sujets d'actualité, par exemple la politique de Frédéric II, des questions sur des rites religieux ou des fêtes contemporaines, des découvertes scientifiques comme celle des frères Montgolfier. En outre, des cours transmettant des « connaissances utiles pour le citoyen " ("nützliche Bürgerkenntnisse ») sont dispensés. Les élèves y apprennent entre autres à se familiariser avec la constitution prussienne, les différentes institutions et les conseils à l'échelle nationale et locale ${ }^{34}$. Des enseignements plus spécifiques et optionnels qui s'orientent déjà vers une formation pré-professionnelle sont également dispensés : il s’agit par exemple de leçons de mathématiques appliquées pour les élèves souhaitant s'orienter vers le commerce.

\section{Conclusion}

Le philanthropinisme et le néohumanisme ne sont pas simplement deux courants pédagogiques opposés qui se succèdent dans l'histoire de l'éducation. Les débats éducatifs des années autour de 1800 montrent qu'ils sont aussi contemporains et qu'une querelle des philanthropinistes et des néohumanistes a bel et bien existé. Même si on peut effectivement parler d'une relation antagoniste entre ces deux courants éducatifs, il faut relativiser une telle représentation et considérer les points de convergence de ces deux camps pédagogiques. La nature de leur relation est donc plus complexe qu'il n'y parait au premier abord et que l'image donnée par l'historiographie. Mais surtout, il est intéressant de s'arrêter sur la tentative de synthèse de Friedrich Gedike. Ce dernier a su concilier les fins éducatives, mais aussi

gehören. Nur diese allein scheinen mir dem sinnlichen Anfänger fasslich und unterhaltend. Alle Abstraktion ermüdet ihn. Fasslichkeit, Simplizität, praktisches Interesse und Mannigfaltigkeit waren die Regeln, die ich mir selbst bei der Sammlung meines griechischen, lateinischen und französischen Lesebuches vorschrieb. " (Friedrich Gedike, Einige Gedanken über Schulbücher und Jugendschriften (1787), in F. G., Gesammlete Schulschriften, vol. 1, Berlin, 1785, p. 78).

34 Friedrich Gedike, Praktischer Beitrag zur Methodik des öffentlichen Unterrichts (1781), ibid., p. 127. 
les contenus de l'enseignement recommandés par ces deux courants rivaux, en faisant de ses établissements berlinois un lieu à la fois de formation générale, avec pour base les humanités (langues et littératures anciennes), et de formation pratique et en partie "pré-professionnalisante ", formant des citoyens se préparant à servir l'État et leur communauté. Pour ce pédagogue berlinois, une formation générale, intellectuelle et principalement linguistique, visant le développement des différentes facultés de l'homme et le libre développement de la personnalité d'un côté, et une formation plus pratique, orientée vers les choses et le monde contemporain, visant la formation de citoyens travaillant au progrès de l'État de l'autre, ne sont en aucun cas incompatibles. Non seulement elles sont compatibles, mais il est selon lui souhaitable de les articuler au sein de l'école publique. Les deux modèles éducatifs ne forment chez Gedike qu'une seule et même ouvre éducative, tels les deux volets d'un diptyque. Délaisser l'un de ces volets porterait préjudice aux jeunes gens et à l'avenir de la Prusse, et, au-delà, de l'humanité. Telle est la conviction de Gedike, conviction qui l'a guidé dans son action en tant qu'enseignant, directeur de lycée et membre du conseil de l'éducation de Prusse.

\author{
Mathilde Lerenard \\ EA $4574 \mathrm{SPH}$ \\ Université Bordeaux Montaigne \\ mathilde.lerenard@u-bordeaux-montaigne.fr
}

\begin{abstract}
Résumé
Les années 1800 en Allemagne sont particulièrement riches en réformes scolaires et en débats sur l'éducation. À travers ce foisonnement d'idées et de pratiques nouvelles, deux courants se distinguent à la fin du XVIII ${ }^{e}$ siècle par les objectifs qu'ils assignent à l'éducation et les modalités d'enseignement qu'ils préconisent : le philanthropinisme et le néohumanisme. Faut-il former l'homme, dans son individualité, afin qu'il développe ses facultés de manière harmonieuse, ou plutôt le citoyen, pour en faire un membre utile de la communauté ? Faut-il favoriser un enseignement formel et linguistique ou au contraire scientifique, axé sur la transmission de connaissances utiles à la vie quotidienne ? L'historiographie a jusqu'ici présenté ces deux courants pédagogiques dans une perspective chronologique, désignant le philanthropinisme comme la pédagogie de l'Aufklärung et le néohumanisme comme celle du début du XIX ${ }^{e}$ siècle, de la deutsche Klassik, et dans une perspective de stricte opposition. La démarche choisie ici, visant à nuancer cette thèse, consiste à revenir sur la période charnière de 1800 et sur la querelle qui éclate entre philanthropinistes et néohumanistes en adoptant une perspective comparative. Le cas du pédagogue Friedrich Gedike et de ses établissements scolaires berlinois démontre en effet que la relation entre ces deux systèmes pédagogiques concurrents est plus complexe qu'une simple opposition symétrique et fait figure d'exemple en matière de conciliation de ces deux courants.
\end{abstract}

\title{
Mots-clés
}

Éducation, Allemagne,1800, philanthropinisme, néohumanisme, Friedrich Gedike.

\footnotetext{
Absract

The period around 1800 in Germany produced important changes in education and educational theory. Among these many thoughts on education and new practices in the late $18^{\text {th }}$ century, there are two main movements which differ on their education's goals and teaching methods: the philan-
} 
thropinism and the new-humanism. Should we educate man in his individuality, in order to develop his powers to a complete and harmonious whole, or rather the citizen, so that he becomes a useful member of the community? Should we emphasize the value of formal and linguistic education or, on the contrary, the value of utilitarian and scientific education? Until now, most studies have analysed these two pedagogical movements as being diametrically opposed, as well as from a chronological viewpoint, designating the philanthropinism as the Enlightenment pedagogy and the new-humanism as the pedagogy of the early $19^{\text {th }}$ century, of the German Klassik. The thought process which has been chosen aims at qualifying this thesis and consists in coming back to this critical period around 1800 and the quarrel which breaks out between philanthropinists and new-humanists by adopting a comparative point of view. The case of the educator Friedrich Gedike and his Berlin schools shows that the relationship between these two competitive educational systems is more complex than a simple symmetrical opposition and represents a model of conciliation between these two movements.

\section{Keywords}

Education, Germany, 1800, philanthropinism, new-humanism, Friedrich Gedike. 